\title{
An LMI Technique for the Global Stabilization of Nonlinear Polynomial Systems
}

\author{
M. Moez Belhaouane, R. Mtar, H. Belkhiria Ayadi, N. Benhadj Braiek
}

\begin{abstract}
Mohamed Moez Belhaouane, Riadh Mtar, Hela Belkhiria Ayadi, Naceur Benhadj Braiek
Laboratoire d'Etude et Commande Automatique de Processus - LECAP

Ecole Polytechnique de Tunisie (EPT), BP.743, 2078 La Marsa, Tunis, Tunisie.

E-mail: \{moez.belhaouane, riadh.mtar, hela.ayadi, naceur.benhadj\}@ept.rnu.tn
\end{abstract}

\begin{abstract}
This paper deals with the global asymptotic stabilization of nonlinear polynomial systems within the framework of Linear Matrix Inequalities (LMIs). By employing the well-known Lyapunov stability direct method and the Kronecker product properties, we develop a technique of designing a state feedback control law which stabilizes quadratically the studied systems. Our main goal is to derive sufficient LMI stabilization conditions which resolution yields a stabilizing control law of polynomial systems.
\end{abstract}

Keywords: Nonlinear Polynomial systems, Lyapunov method, Global stabilization, Kronecker product, LMI approach.

\section{Introduction}

The control theoretician role may be viewed as one of developing methods that allows the control engineer to make which seems relatively natural and physically motivated $[1,2]$. Generally the main and first object in the control theory is to ensure the stability and the convergence of the considered system. In this context, the problem of stabilization of nonlinear systems has received a great deal of attention and several methods have been proposed in the literature $[3,4,5,6]$. However, the proposed approaches remain restrictive to particular classes of nonlinear models, and there is no general method for the analysis or synthesis of general nonlinear systems. That is the reason of continuing research on study and control of nonlinear systems. The polynomial systems constitute an important class of nonlinear systems which has the advantage to describe the dynamical behavior of a large set of processes as electrical machines and robot manipulators and has also the ability to approach any analytical nonlinear system, since any analytical nonlinear function can be approximated by a polynomial expansion. Let'us note that a lot of works have considered the modeling, analysis and control of the polynomial systems $[7,8,9,10,11,12,13,14,15,16]$. The main key of these developments is the description of the polynomial system by using the Kronecker power of the state vector [17].

In the other hand, Linear Matrix Inequalities (LMIs) have emerged as a powerful formulation and design technique for a variety of control problems $[18,19,20]$. Since solving LMI's is a convex optimization problem, such formulation offer a numerically tractable means of attacked problems that lack an analytical solution. Besides, efficient interior-point algorithms are now available to solve the generic LMI problems. They are applied to several important process control applications including control structure selection, robust controller analysis and design, and optimal design of experiments [21, 22, 23]. Consequently, reducing a control design problem to an LMI can be considered as a practical solution [24].

The contribution of the present paper consists on the use of the Lyapunov method with a quadratic candidate function, to derive a practice sufficient condition ensuring the global asymptotic stabilization of the original equilibrium of a polynomial system. This condition is then reformulated in the form of an 
LMI feasibility problem which can be solved using the numerical software as MATLAB.

This paper is organized as follows: In section 2 the description of the studied systems and necessary mathematical notations are introduced. Then, in the next section, the problem of stabilizing control law synthesis of polynomial systems is investigated. The section 4 proposes an LMI formulation of the obtained stabilization condition. An illustrative example is reported in section 5 to implement the developed approach.

\section{Studied polynomial systems and Mathematical Notations}

\subsection{Studied polynomial systems}

The studied nonlinear polynomial systems are described by the following state equation:

$$
\dot{X}=f(X)+G U,
$$

where $f(X)$ is a vectorial polynomial function of $X$.

$$
f(X)=\sum_{i=1}^{r} F_{i} X^{[i]}=\sum_{i=1}^{r} \tilde{F}_{i} \tilde{X}^{[i]}
$$

with

$X=\left[x_{1}, \ldots, x_{n}\right]^{T} \in \mathbb{R}^{n}$,

$$
\left\{\begin{array}{l}
X^{[0]}=1 \\
X^{[i]}=X^{[i-1]} \otimes X=X \otimes X^{[i-1]} \quad \text { for } \quad i \geq 1
\end{array}\right.
$$

$\otimes$ is the symbol of the Kronecker product [17].

$\tilde{X}_{i=1, \ldots, r}^{[i]} \in \mathbb{R}^{n_{i}}$ where $n_{i}=\left(\begin{array}{c}n+i-1 \\ i\end{array}\right)$, is the nun-redundant Kronecker power of the state vector $X$ defined as

$$
\begin{aligned}
& \tilde{X}^{[1]}=X^{[1]}=X, \\
& \forall i \geq 2, \tilde{X}^{[i]}=\left[x_{1}^{i}, x_{1}^{i-1} x_{2}, \ldots, x_{1}^{i-1} x_{n}, \ldots, x_{1}^{i-2} x_{n}^{2}, \ldots, x_{1}^{i-3} x_{2}^{3}, \ldots, x_{n}^{i}\right]^{T},
\end{aligned}
$$

i.e., the components of $\tilde{X}^{[i]}$ are the same that those of $X^{[i]}$ with omission of the repeated terms.

$F_{i, i=1, \ldots, r} \in \mathbb{R}^{n \times n^{i}}$ (resp. $\tilde{F}_{i} \in \mathbb{R}^{n \times n_{i}}$ ) are constant matrices.

The polynomial order $r$ is considered odd: $r=2 s-1$, with $s \in \mathbb{N}^{*}$.

$U \in \mathbb{R}^{m}$ is the input vector and $G$ is a constant $(n \times m)$ matrix.

\subsection{Notations}

In this section, we introduce some useful notations and needed rules and functions. Let the matrices and vectors of the following dimensions

$$
A(p \times q), B(r \times s), C(q \times f), X(n \times 1) \in \mathbb{R}^{n}, Y(m \times 1) \in \mathbb{R}^{m} .
$$

(i) We consider the following notations: $I_{n}:(n \times n)$ identity matrix; $\mathrm{o}_{n \times m}:(n \times m)$ zero matrix; o: zero matrix of convenient dimension; $A^{T}$ : transpose of matrix $\mathrm{A} ; A>\mathrm{o}(A \geq 0)$ : symmetric positive definite (semi-definite) matrix; $e_{k}^{q}: q$ dimensional unit vector which has 1 in the $k^{\text {th }}$ element and zero elsewhere. 
(ii) The relation between the redundant and the nun-redundant Kronecker power of the state vector $X$ can be stated as follows

$$
\left\{\begin{aligned}
\forall i \in \mathbb{N} \exists ! \quad T_{i} & \in \mathbb{R}^{n^{i} \times n_{i}}, \\
X^{[i]} & =T_{i} \tilde{X}^{[i]}
\end{aligned}\right.
$$

A procedure of the determination of the matrix $T_{i}$ is given in [25].

(iii) The permutation matrix denoted $U_{n \times m}$ is defined as

$$
U_{n \times m}=\sum_{i=1}^{n} \sum_{k=1}^{m}\left(e_{i}^{n} \cdot\left(e_{k}^{m}\right)^{T}\right) \otimes\left(e_{k}^{m} \cdot\left(e_{i}^{n}\right)^{T}\right) .
$$

This matrix is square $(n m \times n m)$ and has precisely a single 1 in each row and in each column. Among the main properties of this matrix presented in [17], [11], we recall the following useful ones

$$
\begin{gathered}
(B \otimes A)=U_{r \times p}(A \otimes B) U_{q \times s}, \\
(X \otimes Y)=U_{n \times m}(Y \otimes X), \\
\forall i \leq k \quad X^{[k]}=U_{n^{i} \times n^{k-i}} X^{[k]} .
\end{gathered}
$$

(iv) An important vector valued function of matrix denoted vec(.) was defined in [17] as follows

$$
A=\left[\begin{array}{llll}
c_{1} & c_{2} & \ldots & c_{q}
\end{array}\right] \in \mathbb{R}^{p \times q},
$$

where

$$
\begin{gathered}
\forall i \in\{1, \ldots, q\}, c_{i} \in \mathbb{R}^{p} \text { are the columns of } \mathrm{A} \\
\qquad \operatorname{vec}(A)=\left[\begin{array}{llll}
c_{1}^{T} & c_{2}^{T} & \ldots & c_{q}^{T}
\end{array}\right]^{T} \in \mathbb{R}^{p q} .
\end{gathered}
$$

We recall the following useful rules [17] of this function

$$
\begin{gathered}
\operatorname{vec}(B A C)=\left(C^{T} \otimes B\right) \operatorname{vec}(A), \\
\operatorname{vec}\left(A^{T}\right)=U_{p \times q} \operatorname{vec}(A) .
\end{gathered}
$$

(v) A special function $\operatorname{mat}_{(n, m)}($.$) can be defined as follows$

if $V$ is a vector of dimension $p=n . m$ then $M=\operatorname{mat}_{(n, m)}(V)$ is the $(n \times m)$ matrix verifying $V=\operatorname{vec}(M)$.

(vi) For a polynomial vectorial function

$$
a(X)=\sum_{i=1}^{r} A_{i} X^{[i]},
$$

where $X \in \mathbb{R}^{n}$ and $A_{i}$ are $\left(n \times n^{i}\right)$ constant matrices, we define the $(v \times v)$ matrix $\mathcal{M}(a)$ as

$$
\mathcal{M}(a)=\left[\begin{array}{ccccc}
M_{11}\left(A_{1}\right) & M_{12}\left(A_{2}\right) & 0 & \ldots & 0 \\
0 & M_{22}\left(A_{3}\right) & \ddots & \ddots & \vdots \\
\vdots & \ddots & \ddots & \ddots & 0 \\
\vdots & & \ddots & M_{s-1, s-1}\left(A_{2 s-3}\right) & M_{s-1, s}\left(A_{2 s-2}\right) \\
0 & \ldots & \ldots & 0 & M_{s, s}\left(A_{2 s-1}\right)
\end{array}\right]
$$


with $v=n+n^{2}+\ldots+n^{s}$.

- For $j=1, \ldots, s$

$$
M_{j, j}\left(A_{2 j-1}\right)=\left[\begin{array}{c}
\operatorname{mat}_{\left(n^{j-1}, n^{j}\right)}\left(A_{2 j-1}^{1 T}\right) \\
\operatorname{mat}_{\left(n^{j-1}, n^{j}\right)}\left(A_{2 j-1}^{2 T}\right) \\
\vdots \\
\operatorname{mat}_{\left(n^{j-1}, n^{j}\right)}\left(A_{2 j-1}^{n T}\right)
\end{array}\right],
$$

- For $j=1, \ldots, s-1$

$$
M_{j, j+1}\left(A_{2 j}\right)=\left[\begin{array}{c}
\operatorname{mat}_{\left(n^{j-1}, n^{j}\right)}\left(A_{2 j}^{1 T}\right) \\
\operatorname{mat}_{\left(n^{j-1}, n^{j}\right)}\left(A_{2 j}^{2 T}\right) \\
\vdots \\
\operatorname{mat}_{\left(n^{j-1}, n^{j}\right)}\left(A_{2 j}^{n T}\right)
\end{array}\right],
$$

where $A_{k}^{i}$ is the $i^{t h}$ row of the matrix $A_{k}$

$$
A_{k}=\left[\begin{array}{llll}
A_{k}^{1 T} & A_{k}^{2 T} & \ldots & A_{k}^{n T}
\end{array}\right]^{T} .
$$

(vii) We introduce the matrix $\mathcal{R}$ defined by

$$
\mathcal{R}=\tau_{1}^{+[2]} \cdot \mathcal{U} \cdot \mathcal{H} \cdot \tau_{2},
$$

where

$$
\tau_{1}=\operatorname{Diag}\left(T_{i, i=1, \ldots, S}\right),
$$

with $\tau_{1}^{+}$is the Moore-Penrose pseudo-inverse of $\tau_{1}$ and $\tau_{1}^{+{ }^{[2]}}=\tau_{1}^{+} \otimes \tau_{1}^{+}$.

$$
\begin{gathered}
\tau_{2}=\operatorname{Diag}\left(T_{j, j=2, \ldots, 2 s}\right), \\
\mathcal{U}=\operatorname{Diag}\left(U_{n^{i, i=1, \ldots, s} \times \eta_{0}}\right),
\end{gathered}
$$

$$
\mathcal{H}=\left[\begin{array}{lcccc}
I_{\eta_{1}} & & & & 0 \\
0 \eta_{\eta_{2} \times \eta_{1}} & I_{\eta_{2}} & & & \\
0_{\eta_{3} \times\left(\eta_{1}+\eta_{2}\right)} & & I_{\eta_{3}} & & \\
\vdots & & & \ddots & \\
\mathrm{o}_{\eta_{s} \times\left(\eta_{1}+\eta_{2}+\ldots+\eta_{s-1}\right)} & & & & I_{\eta_{s}}
\end{array}\right],
$$

for $j=0, \ldots . ., s: \eta_{j}=n^{j} \cdot\left(\sum_{i=1}^{s} n^{i}\right)$.

We note $\Gamma$ the matrix defined by

$$
\Gamma=\left(I_{\eta^{2}}+U_{\eta \times \eta}\right)\left(\mathcal{R}^{+T} \mathcal{R}^{T}-I_{\eta^{2}}\right),
$$


with $\eta=\sum_{j=1}^{s} n_{j}=\sum_{j=1}^{s}\left(\begin{array}{c}n+j-1 \\ j\end{array}\right)$ and $\mathcal{R}^{+}$is the Moore-Penrose pseudo-inverse of $\mathcal{R}$.

$$
\beta=\operatorname{rank}(\Gamma)
$$

and $C_{i, i=1, \ldots, \beta}$ are $\beta$ linearly independent columns of $\Gamma$.

(iix) For a $(n \times l)$ matrix $\phi$, we define $\mathcal{D}_{s}(\phi)$ the $(v \times v)$ matrix defined as

$$
\mathcal{D}_{s}(\phi)=\left[\begin{array}{llll}
\phi & & & 0 \\
& \phi \otimes I_{n} & & \\
& & \ddots & \\
& 0 & & \phi \otimes I_{n^{s-1}}
\end{array}\right] .
$$

In the case where the matrix $\phi$ is square $(l=n)$, the matrix $\mathcal{D}_{s}(\phi)$ is also square $(v \times v)$, with $v$ is defined in (vi). As well, if $\phi$ is square and is symmetric positive definite, then so is $\mathcal{D}_{s}(\phi)$.

\section{The Proposed Global Stabilization Condition of Controlled Polynomial Systems}

We consider the polynomial nonlinear systems defined by the equation (1). Our purpose is to determine a polynomial feedback control law

$$
U=k(X)=\sum_{i=1}^{r} K_{i} X^{[i]},
$$

with $K_{i, i=1, \ldots, r}$ are constant gains matrices which stabilizes asymptotically and globally the equilibrium $(X=0)$ of the considered system.

Applying this control law to the open-loop system (1), one obtains the closed-loop system

$$
\begin{aligned}
\dot{X} & =a(X)=(f+G k)(X), \\
& =\sum_{i=1}^{r} A_{i} X^{[i]}
\end{aligned}
$$

where

$$
A_{i}=F_{i}+G K_{i} .
$$

Using a quadratic Lyapunov function $V(X)$ and computing the derivative $\dot{V}(X)$, lead to the sufficient condition of the global asymptotic stabilization of the polynomial system, given by the following theorem 1 .

Theorem 1. The nonlinear polynomial system defined by the equation (1) is globally stabilized by the control law (25), if there exist

- an $(n \times n)$-symmetric positive definite matrix $P$;

- arbitrary parameters $\mu_{i, i=1, \ldots, \beta} \in \mathbb{R}$;

- gain matrices $K_{i, i=1, \ldots, r}$; 
such that the $(\eta \times \eta)$ symmetric matrix $Q$ defined by

$$
\begin{aligned}
\mathcal{Q} & =\tau_{1}^{T}\left[\mathcal{D}_{S}(P) \mathcal{M}(f)+\mathcal{M}(f)^{T} \mathcal{D}_{S}(P)\right] \tau_{1}+\tau_{1}^{T}\left[\mathcal{D}_{S}(P) \mathcal{G N}(k)+\left(\mathcal{D}_{S}(P) \operatorname{G\mathcal {N}}(k)\right)^{T}\right] \tau_{1} \\
& +\sum_{i=1}^{\beta} \mu_{i} \operatorname{mat}_{(\eta, \eta)}\left(C_{i}\right),
\end{aligned}
$$

be negative definite.

Where $\beta$ and $C_{i, i=1, \ldots, \beta}$ are defined in (23).

Proof. Consider the quadratic Lyapunov function

$$
V(X)=X^{T} P X,
$$

Differentiating $V(X)$ along the trajectory of the system (26), we obtains

$$
\begin{aligned}
\dot{V}(X) & =\sum_{k=1}^{r}\left(X^{T} P A_{k} X^{[k]}+X^{[k]^{T}} A_{k}^{T} P X\right), \\
& =2 \sum_{k=1}^{r} X^{T} P A_{k} X^{[k]} .
\end{aligned}
$$

Using the rule of the vec-function (10), the relation (30) can be written as

$$
\dot{V}(X)=2 \sum_{k=1}^{r} V_{k}^{T} X^{[k+1]}
$$

where

$$
V_{k}=\operatorname{vec}\left(P A_{k}\right) .
$$

We can write

$$
\sum_{k=1}^{r=2 s-1} V_{k}^{T} X^{[k+1]}=\sum_{j=1}^{s-1} V_{2 j}^{T} X^{[2 j+1]}+\sum_{j=1}^{s} V_{2 j-1}^{T} X^{[2 j]},
$$

using the mat-function defined in section 2 , one has

$$
\dot{V}(X)=2\left[\sum_{j=1}^{s-1} X^{[j]^{T}} \operatorname{mat}_{\left(n^{j}, n^{j+1}\right)}\left(V_{2 j}^{T}\right) X^{[j+1]}+\sum_{j=1}^{s} X^{[j]^{T}} \operatorname{mat}_{\left(n^{j}, n^{j}\right)}\left(V_{2 j-1}^{T}\right) X^{[j]}\right] .
$$

Applying the following lemma [11]

Lemma 2. Consider a $\left(n \times n^{k}\right)$ matrix $A(k \in \mathbb{N})$ and $a(n \times n)$ matrix $P$.

Let $i$ and $j$ two integers verifying $i+j=k+1$ and $i \geq 1$. Then

$$
\operatorname{mat}_{\left(n^{i}, n^{j}\right)}(\operatorname{vec}(P A))=U_{n^{i-1} \times n}\left(P \otimes I_{n^{i-1}}\right) \cdot \mathbb{M},
$$

with

$$
\mathbb{M}=\left[\begin{array}{c}
\operatorname{mat}_{\left(n^{i-1}, n^{j}\right)}\left(A^{1 T}\right) \\
\operatorname{mat}_{\left(n^{i-1}, n^{j}\right)}\left(A^{2 T}\right) \\
\vdots \\
\operatorname{mat}_{\left(n^{i-1}, n^{j}\right)}\left(A^{n T}\right)
\end{array}\right]
$$

where $A^{i}$ denotes the $i^{\text {th }}$ row of the matrix $A$

$$
A=\left[\begin{array}{llll}
A^{1 T} & A^{2 T} & \ldots & A^{n T}
\end{array}\right]^{T}
$$


leads to the following relations

$$
\begin{aligned}
& \operatorname{mat}_{\left(n^{j}, n^{j+1}\right)}\left(V_{2 j}^{T}\right)=U_{n^{j-1} \times n}\left(P \otimes I_{n^{j-1}}\right) M_{j, j+1}\left(A_{2 j}\right), \\
& \operatorname{mat}_{\left(n^{j}, n^{j}\right)}\left(V_{2 j-1}^{T}\right)=U_{n^{j-1} \times n}\left(P \otimes I_{n^{j-1}}\right) M_{j, j}\left(A_{2 j-1}\right),
\end{aligned}
$$

where $M_{j, j+1}\left(A_{2 j}\right)$ and $M_{j, j}\left(A_{2 j-1}\right)$ are defined respectively in (15) and (14) and $U_{n^{j-1} \times n}$ is mentioned in (20).

Using the results (35) and (36), the equality (34) can be expressed as

$$
\begin{aligned}
\dot{V}(X) & =2\left[\sum_{j=1}^{s-1} X^{[j]^{T}} U_{n^{j-1} \times n}\left(P \otimes I_{n^{j-1}}\right) M_{j, j+1}\left(A_{2 j}\right) X^{[j+1]}\right. \\
& \left.+\sum_{j=1}^{s} X^{[j]^{T}} U_{n^{j-1} \times n}\left(P \otimes I_{n^{j-1}}\right) M_{j, j}\left(A_{2 j-1}\right) X^{[j]}\right],
\end{aligned}
$$

by means of the relation (9), one obtains

$$
\dot{V}(X)=2\left[\sum_{j=1}^{s-1} X^{[j]^{T}}\left(P \otimes I_{n^{j-1}}\right) M_{j, j+1}\left(A_{2 j}\right) X^{[j+1]}+\sum_{j=1}^{s} X^{[j]^{T}}\left(P \otimes I_{n^{j-1}}\right) M_{j, j}\left(A_{2 j-1}\right) X^{[j]}\right] .
$$

Consequently, we obtain

$$
\begin{aligned}
\dot{V}(X) & ={ }_{2} X^{T} \mathcal{D}_{S}(P) \mathcal{M}(a) X \\
& =X^{T}\left(\mathcal{D}_{S}(P) \mathcal{M}(a)+\mathcal{N}(a)^{T} \mathcal{D}_{S}(P)\right) X
\end{aligned}
$$

with

$$
X=\left[\begin{array}{llll}
X^{T} & X^{[2]^{T}} & \cdots & X^{[s]^{T}}
\end{array}\right]^{T}
$$

$\mathcal{D}_{S}(P)$ and $\mathcal{M}(a)$ are defined respectively in (24) and (13).

Using the nun-redundant Kronecker product power form, the vector $\mathcal{X}$ can be written as

$$
x=\tau_{1} \tilde{x}
$$

where $\widetilde{X}=\left[\begin{array}{llll}\widetilde{X}^{T} & \widetilde{X}^{[2]^{T}} & \ldots & \widetilde{X}^{[s]^{T}}\end{array}\right]^{T} \in \mathbb{R}^{\eta}, \eta=\sum_{j=1}^{s} n_{j}$ and $\tau_{1}$ is defined in (18).

Then $\dot{V}(X)$ can be written in the following form

$$
\dot{V}(X)=\widetilde{X}^{T} \tau_{1}^{T}\left(\mathcal{D}_{S}(P) \mathcal{M}(a)+\mathcal{M}(a)^{T} \mathcal{D}_{S}(P)\right) \tau_{1} \widetilde{X},
$$

A sufficient condition of the global asymptotic stability of the equilibrium $(X=0)$ is that the quadratic form $\dot{V}(X)$ is negative definite. This condition can be ensured if there exists a symmetric negative definite $Q \in \mathbb{R}^{\eta \times \eta}$ such that

$$
\widetilde{X}^{T} \tau_{1}^{T}\left(\mathcal{D}_{S}(P) \mathcal{M}(a)+\mathcal{M}(a)^{T} \mathcal{D}_{S}(P)\right) \tau_{1} \widetilde{X}=\widetilde{X}^{T} \mathcal{Q} \widetilde{X},
$$

using the vec-function, the equality (43) can be expressed as

$$
\operatorname{vec}^{T}\left(\mathcal{Q}-\tau_{1}^{T}\left(\mathcal{D}_{S}(P) \mathcal{M}(a)+\mathcal{M}(a)^{T} \mathcal{D}_{S}(P)\right) \tau_{1}\right) \widetilde{\mathcal{X}}^{[2]}=0 .
$$

But, it can be easily checked that $\widetilde{X}^{[2]}$ can be written as

$$
\widetilde{X}^{[2]}=\mathcal{R} \widetilde{X}_{2},
$$


where

$$
\widetilde{X}_{2}=\left[\begin{array}{llllll}
\widetilde{X}^{[2] T} & \ldots & \widetilde{X}^{[s+1] T} & \widetilde{X}^{[s+2] T} & \ldots & \widetilde{X}^{[2 s] T}
\end{array}\right]^{T},
$$

and $\mathcal{R}$ is the matrix defined in (17). The proof of the relation (45) is given in [11].

Therefore the equality (44) yields the following equation

$$
\mathcal{R}^{T} \operatorname{vec}(S)=\mathrm{o},
$$

with: $S=\mathcal{Q}-\tau_{1}^{T}\left(\mathcal{D}_{S}(P) \mathcal{M}(a)+\mathcal{M}(a)^{T} \mathcal{D}_{S}(P)\right) \tau_{1}$.

The $\eta^{2}$-vector vec $(S)$ solution of (47) can be expressed as

$$
\operatorname{vec}(S)=\left(\mathcal{R}^{+T} \mathcal{R}^{T}-I_{\eta^{2}}\right) y,
$$

where $y$ is an arbitrary vector of $\mathbb{R}^{\eta^{2}}$.

The matrix $S$ is symmetric since $Q$ is symmetric, then we can write

$$
S=\frac{1}{2}\left(S+S^{T}\right),
$$

and using the property (11) yields

$$
\operatorname{vec}(S)=\frac{1}{2}\left(I_{\eta^{2}}+U_{\eta \times \eta}\right) \operatorname{vec}(S)=\sum_{i=1}^{\beta} \mu_{i} C_{i},
$$

where

- $\beta=\operatorname{rank}\left[\left(I_{\eta^{2}}+U_{\eta \times \eta}\right)\left(\mathcal{R}^{+T} \mathcal{R}^{T}-I_{\eta^{2}}\right)\right]$,

- $C_{i, i=1, \ldots, \beta}$ are $\beta$ linearly independent columns of

$$
\left(I_{\eta^{2}}+U_{\eta \times \eta}\right)\left(\mathcal{R}^{+T} \mathcal{R}^{T}-I_{\eta^{2}}\right),
$$

- $\mu_{i, i=1, \ldots, \beta}$ are arbitrary values.

Consequently, the symmetric matrix $Q$ verifying (47) is of the following form

$$
\mathcal{Q}=\tau_{1}^{T}\left(\mathcal{D}_{S}(P) \mathcal{M}(a)+\mathcal{M}(a)^{T} \mathcal{D}_{S}(P)\right) \tau_{1}+\sum_{i=1}^{\beta} \mu_{i} \operatorname{mat}_{(\eta, \eta)}\left(C_{i}\right) .
$$

According to (26) and the following lemma [10]

Lemma 3. Let $G \in \mathbb{R}^{n \times m}, k($.$) a polynomial vectorial function defined in (25) and G . k($.$) the resultant$ product of $G$ by $k($.$) , then one has$

$$
\mathcal{M}(G . k)=\operatorname{G\mathcal {N}}(k),
$$

where $\mathcal{G}=D_{s}(G)$ and $\mathcal{M}($.$) the matrix function defined in (13).$

Thus, we can write

$$
\mathcal{M}(a)=\mathcal{M}(f+G k)=\mathcal{M}(f)+\operatorname{G\mathcal {N}}(k),
$$

finally, we obtain the following quadratic form of the symmetric matrix $Q$

$$
\begin{aligned}
\mathcal{Q} & =\tau_{1}^{T}\left[\mathcal{D}_{S}(P) \mathcal{M}(f)+\mathcal{M}(f)^{T} \mathcal{D}_{S}(P)\right] \tau_{1}+\tau_{1}^{T}\left[\mathcal{D}_{S}(P) \mathcal{G} \mathcal{M}(k)+\mathcal{M}(k)^{T} \mathcal{G}^{T} \mathcal{D}_{S}(P)\right] \tau_{1} \\
& +\sum_{i=1}^{\beta} \mu_{i} m a t_{(\eta, \eta)}\left(C_{i}\right) .
\end{aligned}
$$

If $Q$ is negative definite, then the derivative $\dot{V}(X)$ is negative definite.

Which ends the proof. 


\section{Stabilizing Control Synthesis using the LMI approach}

In this section we show how the stabilization problem stated by the theorem 1 can be formulated as an LMI feasibility problem.

Let recall that our main problem is to find

- gain matrices $K_{i, i=1, \ldots, r}$;

- a $(n \times n)$ matrix $P$;

- real parameters $\mu_{i, i=1, \ldots, \beta}$;

such that

$$
\begin{gathered}
P>0, \\
\tau_{1}^{T}\left[\mathcal{D}_{S}(P) \mathcal{M}(f)+\mathcal{M}(f)^{T} \mathcal{D}_{S}(P)\right] \tau_{1}+\tau_{1}^{T}\left[\mathcal{D}_{S}(P) \mathcal{G M}(k)+\mathcal{M}(k)^{T} \mathcal{G}^{T} \mathcal{D}_{S}(P)\right] \tau_{1} \\
+\sum_{i=1}^{\beta} \mu_{i} \operatorname{mat}_{(\eta, \eta)}\left(C_{i}\right)<0 .
\end{gathered}
$$

Note that this problem is nonlinear with respect of the unknown parameters $P, K_{i}$ and $\mu_{i}$, since the inequality (56) is bilinear on $\left(P, K_{i}\right)$. To overcome this problem we make use of the known Schur's complement [18] and we exploit the separation lemma [26]. In this sequel we transform the BMI problem into LMI problem as it is shown in the following development.

Making use of the following separation lemma [26]

Lemma 4. For any matrices $A$ and $B$ with appropriate dimensions and for any positive scalar $\varepsilon>0$, one has:

$$
A^{T} B+B^{T} A \leq \varepsilon A^{T} A+\varepsilon^{-1} B^{T} B,
$$

one obtains

$$
\begin{aligned}
& \mathcal{Q} \leq \tau_{1}^{T}\left[\mathcal{D}_{S}(P) \mathcal{M}(f)+\mathcal{M}(f)^{T} \mathcal{D}_{S}(P)\right] \tau_{1}+\sum_{i=1}^{\beta} \mu_{i} \text { mat }_{(\eta, \eta)}\left(C_{i}\right) \\
& +\gamma \tau_{1}^{T} \mathcal{D}_{S}(P)^{T} \mathcal{D}_{S}(P) \tau_{1}+\gamma^{-1} \tau_{1}^{T} \mathcal{M}(k)^{T} \mathcal{G}^{T} \mathcal{G \mathcal { M }}(k) \tau_{1},
\end{aligned}
$$

with $\gamma>0$.

Then, to ensure that the matrix $Q$ is negative definite, it is sufficient to have

$$
\begin{aligned}
& \tau_{1}^{T}\left[\mathcal{D}_{S}(P) \mathcal{M}(f)+\mathcal{M}(f)^{T} \mathcal{D}_{S}(P)\right] \tau_{1}+\sum_{i=1}^{\beta} \mu_{i} m a t_{(\eta, \eta)}\left(C_{i}\right) \\
& -\tau_{1}^{T} \mathcal{D}_{S}(P)^{T}(-\gamma I) \mathcal{D}_{S}(P) \tau_{1}-\tau_{1}^{T} \mathcal{M}(k)^{T} \mathcal{G}^{T}\left(-\gamma^{-1} I\right) \mathcal{G M}(k) \tau_{1}<0 .
\end{aligned}
$$

Using the Generalized Schur's complement, the inequality (58) is equivalent to

$$
\left[\begin{array}{ccc}
\tau_{1}^{T}\left(\mathcal{D}_{S}(P) \mathcal{M}(f)+\mathcal{M}(f)^{T} \mathcal{D}_{S}(P)\right) \tau_{1}+\sum_{i=1}^{\beta} \mu_{i} \operatorname{mat}_{(\eta, \eta)}\left(C_{i}\right) & \left(\mathcal{D}_{S}(P) \tau_{1}\right)^{T} & \left(\operatorname{G\mathcal {M}}(k) \tau_{1}\right)^{T} \\
\mathcal{D}_{S}(P) \tau_{1} & -\gamma^{-1} I & 0 \\
\mathcal{G \mathcal { M }}(k) \tau_{1} & 0 & -\gamma I
\end{array}\right]<0,
$$

when pre-and post-multiplying the inequality (59) by $\Xi=\operatorname{diag}\left(I, I, \gamma^{-1} I\right)$, we get

$$
\left[\begin{array}{ccc}
\tau_{1}^{T}\left(\mathcal{D}_{S}(P) \mathcal{M}(f)+\mathcal{M}(f)^{T} \mathcal{D}_{S}(P)\right) \tau_{1}+\sum_{i=1}^{\beta} \mu_{i} m a t_{(\eta, \eta)}\left(C_{i}\right) & \left(\mathcal{D}_{S}(P) \tau_{1}\right)^{T} & \left(\mathcal{G W}(k) \tau_{1}\right)^{T} \\
\mathcal{D}_{S}(P) \tau_{1} & -\gamma^{-1} I & 0 \\
\mathcal{G W}(k) \tau_{1} & 0 & -\gamma^{-1} I
\end{array}\right]<0
$$

with $\mathcal{W}(k)=\gamma^{-1} \mathcal{M}(k)$.

This new inequality is linear on the decision variables, and then we can state the following result. 
Theorem 5. The equilibrium $(X=0)$ of the system (1) is globally asymptotically stabilizable if there exist

- $a(n \times n)$-symmetric positive definite matrix $P$;

- arbitrary parameters $\mu_{i, i=1, \ldots, \beta} \in \mathbb{R}$;

- gain matrices $K_{i, i=1, \ldots, r}$;

- a real $\gamma>0$;

such that

$$
P>0
$$

and

$$
\left[\begin{array}{ccc}
\tau_{1}^{T}\left(\mathcal{D}_{S}(P) \mathcal{M}(f)+\mathcal{M}(f)^{T} \mathcal{D}_{S}(P)\right) \tau_{1}+\sum_{i=1}^{\beta} \mu_{i} \operatorname{mat}_{(\eta, \eta)}\left(C_{i}\right) & \left(\mathcal{D}_{S}(P) \tau_{1}\right)^{T} & \left(\mathcal{G W}(k) \tau_{1}\right)^{T} \\
\mathcal{D}_{S}(P) \tau_{1} & -\gamma^{-1} I & 0 \\
\mathcal{G W}(k) \tau_{1} & 0 & -\gamma^{-1} I
\end{array}\right]<0
$$

Thus, a stabilizing control law (25) for the considered polynomial system (1) can be characterized by applying the following procedure

1. Solve the LMI feasibility problem i.e., find the matrices $\mathcal{D}_{S}(P), \mathcal{W}(k)$ and the parameters $\mu_{i}$ and $\gamma$ such that the inequalities (61), (62) are verified.

2. Extract the gain matrices $K_{i}$ from the relation $\mathcal{M}(k)=\gamma \mathcal{W}(k)$.

This optimization problem can be carried out using MATLAB software. To provide the effectiveness of the proposed approach, we consider the following numerical example.

\section{Illustrative Example}

Our aim in this section is to apply the proposed approach for the global stabilization of the following polynomial system

$$
\left\{\begin{array}{l}
\dot{x}_{1}=-x_{1}+x_{2}+x_{1}^{2}+x_{1} x_{2}-x_{1}^{3}+x_{1}^{2} x_{2}-x_{1} x_{2}^{2}+2 x_{2}^{3}, \\
\dot{x}_{2}=-x_{1}+1.5 x_{2}-x_{1}^{2}-0.5 x_{1} x_{2}-x_{1}^{3}-x_{1}^{2} x_{2}+0.5 x_{1} x_{2}^{2}-2 x_{2}^{3}+u .
\end{array}\right.
$$

Using the Kronecker product, this system can be described by the following compact state equation

$$
\dot{X}=F_{1} X+F_{2} X^{[2]}+F_{3} X^{[3]}+G U,
$$

with

$$
F_{1}=\left[\begin{array}{cc}
-1 & 1 \\
-1 & 1.5
\end{array}\right] \quad F_{2}=\left[\begin{array}{cccc}
1 & 1 & 0 & 0 \\
-1 & -0.5 & 0 & 0
\end{array}\right] \quad F_{3}=\left[\begin{array}{cccccccc}
-1 & 1 & 0 & -1 & 0 & 0 & 0 & 2 \\
-1 & -1 & 0 & 0.5 & 0 & 0 & 0 & -2
\end{array}\right]
$$

and $G=\left[\begin{array}{c}0 \\ 1\end{array}\right]$.

We are interested with the stabilization of the origin equilibrium $(X=0)$ of the system (64).

Let us note that the uncontrolled $(U=0)$ non linear system is unstable since the matrix $F_{1}$ has an unstable eigenvalue. 
Solving the optimization problem formulated by theorem 2, we obtain

$$
\left\{\begin{array}{l}
\mu_{1}=-0.0319 \\
\mu_{2}=0.0246 \\
\mu_{3}=0.1928
\end{array} \quad ; \quad P=\left[\begin{array}{ll}
0.0246 & 0.0063 \\
0.0063 & 0.0071
\end{array}\right] \quad ; \quad \gamma=2.0240 .\right.
$$

The searched gain matrices, extracted from $\mathcal{M}(k)$, are given by

$$
\begin{aligned}
& K_{1}=\left[\begin{array}{ll}
-9.8872 & -5.5518
\end{array}\right] \quad K_{2}=\left[\begin{array}{llll}
2.1050 & 0.8966 & 0 & 0
\end{array}\right]
\end{aligned}
$$

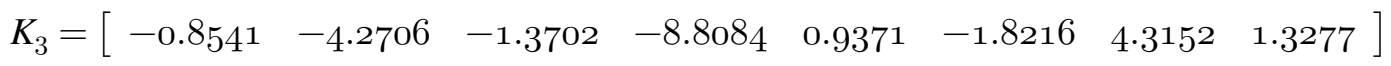

Then a global stabilizing control law can be characterized for the studied system using the previous developed method. This control law can be expressed as

$$
U=K_{1} X+K_{2} X^{[2]}+K_{3} X^{[3]} .
$$

The Figure 1 shows the behavior of the state variables $x_{1}(t)$ and $x_{2}(t)$ of the controlled system from initial conditions which were taken sufficiently far from the initial conditions $\left(x_{1}(0)=-10, x_{2}(0)=10\right)$. It appears that the state variables converge into the origin point which confirm the asymptotic stability of the controlled system.

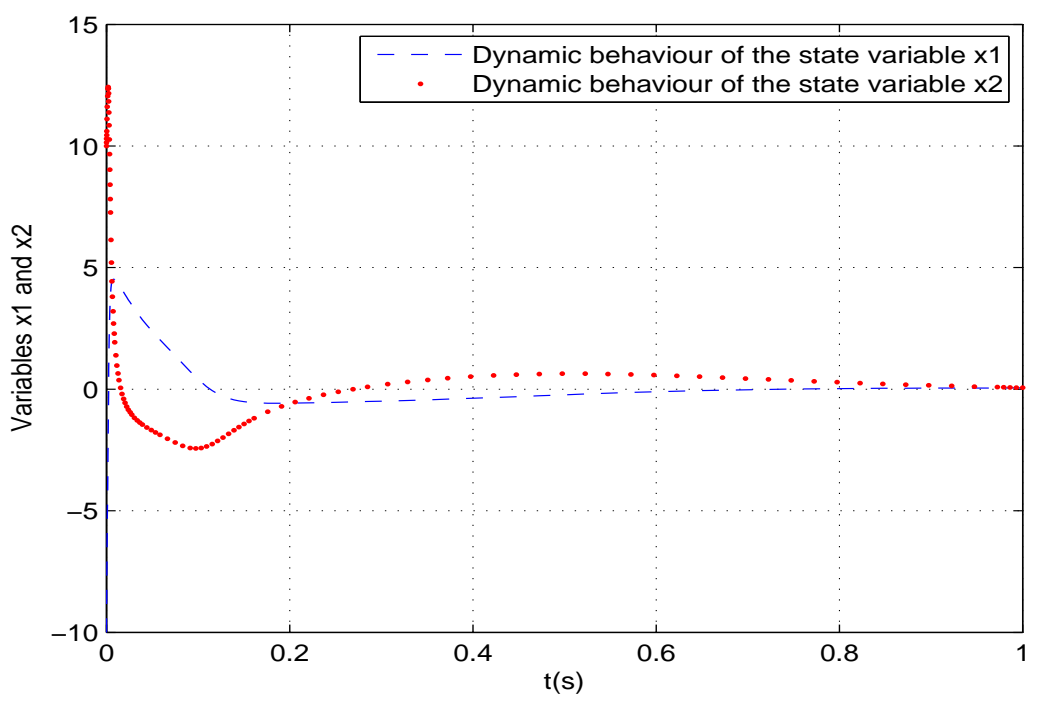

Figure 1: Closed-loop responses of the system (64) with the control law (65).

\section{Conclusion}

In this paper, an original technique has been proposed for the global and asymptotic stabilization of the nonlinear polynomial systems. This new stabilizing approach is based on the Lyapunov direct method and elaborated algebraic developments using the Kronecker product properties. This development has allowed the formulation of the system stabilization condition as an LMI feasibility problem, which resolution leads to a polynomial control law ensuring the quadratic stability in the whole state space of the 
considered system. Further works, will consider extension of these results to the robust stabilization of polynomial uncertain systems.

\section{Bibliography}

[1] J. C. Doyle, "A review of $\mu$ for case studies in robust control," IFAC, pp. 395-402, 1987.

[2] F. Lescher, J. Y. Zhao, and P. Borne, "Switching LPV controllers for a variable speed pitch regulated wind turbine," International Journal of Computers, Communications and Control (IJCCC), vol. 1, no. 4, pp. 73-84, 2006.

[3] A. Isidori, Nonlinear control systems. Springler-verlag, 1995.

[4] P. Borne, J. P. Richard, and N. E. Radhy, "Stability, stabilization, regulation using vector norms," Nonlinear Systems, vol. 2, Stability and stabilization, Chapter 2, pp. 45-90, 1996.

[5] P. Borne, G. Dauphin Tanguy, J. P. Richard, F. Rotella, and I. Zambettakis, Commande et Optimisation des Processus. Technip, 1998.

[6] Y. Blanco, W. Perruquetti, and P. Borne, "Stability and stabilization of nonlinear systems and tanaka-sugeno fuzzy models," European Control Conference ECC'01, 2001.

[7] N. Benhadj Braiek, "On the global stability of nonlinear polynomial systems," IEEE Conference On Decision and Control,CDC'96, December 1996.

[8] N. Benhadj Braiek and F. Rotella, "Design of observers for nonlinear time variant systems," IEEE Syst. Man and Cybernetics Conference, vol. 4, pp. 219-225, 1993.

[9] N. Benhadj Braiek and F. Rotella, "State observer design for analytical nonlinear systems," IEEE Syst. Man and Cybernetics Conference, vol. 3, pp. 2045-2050, October 1994.

[10] N. Benhadj Braiek and F. Rotella, "Stabilization of nonlinear systems using a Kronecker product approach,” European Control Conference ECC’95, pp. 2304-2309, september 1995.

[11] N. Benhadj Braiek, F. Rotella, and M. Benrejeb, "Algebraic criteria for global stability analysis of nonlinear systems," Journal of Systems Analysis Modelling and Simulation, Gordon and Breach Science Publishers, vol. 17, pp. 221-227, 1995.

[12] N. Benhadj Braiek, "Feedback stabilization and stability domain estimation of nonlinear systems," Journal of The Franklin Institute, vol. 332, no. 2, pp. 183-193, March 1995.

[13] F. Rotella and G. Tanguy, "Non linear systems: identification and optimal control," Int.J.Control, vol. 48, no. 2, pp. 525-544, 1988.

[14] H. Bouzaouache and N. Benhadj Braiek, "On guaranteed global exponential stability of polynomial singularly perturbed control systems," International Journal of Computers, Communications and Control (IJCCC), vol. 1, no. 4, pp. 21-34, 2006.

[15] H. Bouzaouache and N. Benhadj Braiek, "On the stability analysis of nonlinear systems using polynomial Lyapunov functions," Mathematics and Computers in Simulation, vol. 76, no. 5-6, pp. 316-329, 2008.

[16] H. B. Ayadi and N. Benhadj Braiek, "Robust control of nonlinear polynomial systems," IEEE Syst. Man and Cybernetics Conference, SMC'02, 6-9 October 2002. 
[17] J. Brewer, "Kronecker product and matrix calculus in system theory," IEEE Trans.Circ.Sys, vol. CAS-25, pp. 722-781, 1978.

[18] S. Boyd, L. Ghaoui, and F. Balakrishnan, "Linear Matrix Inequalities in System and Control Theory," SIAM, 1994.

[19] J. G. V. Antwerp and R. D. Braatz, "A tutorial on linear and bilinear matrix inequalities," Journal of process control, vol. 10, pp. 363-385, 2000.

[20] D. Rosinova and V. Vesely, "Robust PID decentralized controller design using LMI," International Journal of Computers, Communications and Control (IJCCC), May 2007.

[21] A. Nemirovsky and P.Gahinet, "The projective method for solving linear matrix inequalities," In Proc. American Control Conf., 1994.

[22] S. Boyd and L. Vandenberghe, "Introduction to convex optimization with engineering applications," Lecture Notes, Information Systems Laboratory, 1995.

[23] C. Scherer, P. Gahinet, and M. Chilali, "Multiobjective output-feedback control via LMI optimization,” IEEE Transactions on Automatic Control, vol. 42, no. 7, pp. 896-910, July 1997.

[24] Y. Nesterov and A.Nemirovsky, "Interior point polynomial methods in convex programming: Theory and applications," SIAM, pp. 101-127, 1994.

[25] N. Benhadj Braiek and F. Rotella, "Logid: a nonlinear systems identification software," Modelling and Simulation of Systems, Scientific Publishing Co., pp. 211-218, 1990.

[26] Zhou and Khargonedkar, "Robust stabilization of linear systems with norm-bounded time-varying uncertainty," Sys. Contr. Letters, vol. 10, pp. 17-20, 1988.

Mohamed Moez Belhaouane was born in Tunis in 1980. He received the Electrical Engineering bachelor and the Master degree in Automatic Control from École Supérieure des Sciences et Techniques de Tunis (ESSTT), in 2003 and 2005, respectively.

Currently, he is research member of the Processes Study and Automatic Control Laboratory (LECAP) in the Polytechnic School of Tunisia and he is working toward Ph.D. degree in Electrical Engineering. His research interests include analysis and control of nonlinear systems, polynomial systems and robust control.

Riadh Mtar was born in Tunis in 1974. He received his Master degree of Systems Analysis and Numerical Treatment from École Nationale d'ingénieurs de Tunis (ENIT), in 2005. He is currently an contractual Assistant in École Supérieure de Commerce (ESC) and research member of the Processes Study and Automatic Control Laboratory (LECAP) in the Polytechnic School of Tunisia. Actually, he is preparing his Ph.D. dissertation in Electrical Engineering from École Nationale d'ingénieurs de Tunis (ENIT). His research interests include analysis and control of nonlinear polynomial discret systems.

Hela Belkhiria Ayadi received her engineer diplomas from the École Nationale d'ingénieurs de Monastir (ENIM) in 1997 and Master degree of Automatic Control from École supérieure des Sciences et Techniques de Tunis (ESSTT), in 1998. She completed her Ph.D. in Electrical Engineering in 2004 from École Nationale d'ingénieurs de Tunis (ENIT). She is now an assistant professor in École supérieure des Sciences et Techniques de Tunis (ESSTT) and research member of the Processes Study and Automatic Control Laboratory (LECAP) in the Polytechnic School of Tunisia. Her research interests include robust analysis and control of nonlinear uncertain systems. 
Naceur Benhadj Braiek was born in Mahdia, Tunisia, in 1963. He obtained the Master of Electrical Engineers and the Master of Systems Analysis and Numerical Processing, both from École Nationale d'ingénieurs de Tunis (ENIT) in 1987, the Master of Automatic Control from Institut Industriel de Nord (École Centrale de Lille) in 1988, the Ph.D. degree in Automatic Control from Université des Sciences et Techniques de Lille, France, 1990, and the Doctorat d'état in Electrical Engineering in 1995 from École Nationale d'ingénieurs de Tunis (ENIT). Now, he is Professor of Electrical Engineering at the University of Tunis - École supérieure des Sciences et Techniques de Tunis (ESSTT). He is also Director of the Processes Study and Automatic Control Laboratory (LECAP) in the Polytechnic School of Tunisia. His domain of interest is related to the modeling, analysis and control of nonlinear systems with applications on Electrical Processes. 